\title{
HARA 65881
}

\section{A NEW METHOD FOR IN-SITU ELECTRON TEMPERATURE DETERMINATIONS FROM PLASMA WAVE PHENOMENA}

\section{HIROSHI OYA ROBERT F. BENSON}

(NASA-TH-X-65881) A NEW METHOD FOR IN SITO ELECTRON TEMPERATURE DETERMINATIONS FROM

PLASMA HAVE PHENOMENA H. Oya, et al (NASA) Apr. $197215 \mathrm{p}$ CSCL $20 \mathrm{I}$

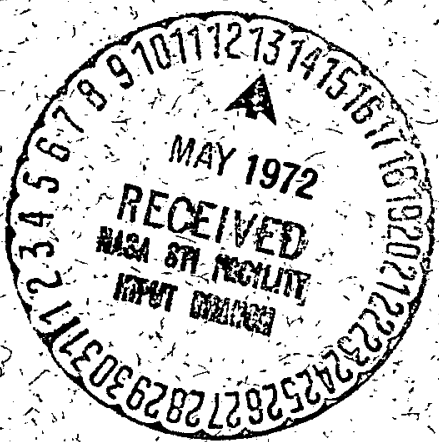




\section{A NEW METHOD FOR IN-SITU ELECTRON TEMPERATURE DETERMINATIONS FROM PLASMA WAVE PHENOMENA}

by

Hiroshi Oya* and Robert F. Benson

\footnotetext{
* Now at the Ionosphere Research Lab., Kyoto University, Kyoto, Japan
} 
A discrepancy has been reported between the values of the electron temperature $\mathrm{T}_{\mathrm{e}}$ deduced from satellite electrostatic probe measurements and ground based radar backscatter measurements [Hanson et al., 1969]. This discrepancy (radar backscatter temperature less than the probe temperature) is not present when the probe experiment is flown on a rocket to lower altitudes [ Brace et al., 1969] but reappears when the rocket probe attains higher altitudes [Brace and McClure, 1971] . There is a need for an independent radio wave method for making in-situ $\mathrm{T}_{\mathrm{e}}$ measurements in order to help resolve this problem. Several methods have been introduced which obtain $T_{e}$ from the periodic amplitude structure often observed during the decay of the $f_{N}$ and $f_{T}$ plasma resonances stimulated by the ionospheric sounders on board the Alouette and ISIS satellites $\left(f_{N}\right.$ is the electron plasma frequency, $f_{T}=\left(f_{N}^{2}+f_{H}^{2}\right)^{1 / 2}$ is the upper-hybrid frequency, where $\mathrm{f}_{\mathrm{H}}$ is the electron cyclotron frequency) [ Warnock et al., 1970 ] (Feldstein and Graff, personal communication, 1971). The main limitations of these methods are that extensive calculations are required and that the electron density scale height must be determined from the ionospheric sounder record (ionogram). This letter presents a new method of determining $\mathrm{T}_{\mathrm{e}}$ from the satellite resonant phenomena. It is based on the "splitting" (observed at high latitudes) of the diffuse resonance which occurs at the frequency $f_{D 1}$ between $f_{H}$ and $2 f_{H}[$ Oya, 1970]. The advantage of this method over the other methods involving ionospheric resonances is the simplicity of the required calculations. There is, however, the limitation of plasma conditions where the $\mathrm{f}_{\mathrm{D} 1}$ resonance can be observed $\left(2 \lesssim \mathrm{f}_{\mathrm{N}} / \mathrm{f}_{\mathrm{H}} \lesssim 3\right)$ and the limitation to mid-to-high latitudes where the "splitting" is observed. 
The diffuse resonances observed on topside ionograms have been interpreted in terms of an electrostatic electron cyclotron harmonic wave instability together with nonlinear wave-wave and nonlinear wave-particle resonant interactions [Oya, 1971]. The instability in this interpretation results from a biMaxwellian electron velocity distribution function, with $\left(\mathrm{T}_{\mathrm{e}}\right)_{\perp} \gg\left(\mathrm{T}_{\mathrm{e}}\right)_{\|}$, which is created by the high-power sounder pulse; the observations indicate that this initial instability persists for no longer than $1.5 \mathrm{msec}\left(\left(\mathrm{T}_{\mathrm{e}}\right)_{\perp}\right.$ and $\left(\mathrm{T}_{\mathrm{e}}\right)_{\|}$refer to the electron temperature corresponding to electron motion perpendicular and parallel to the ambient magnetic field vector $\vec{B}$ ). Fredricks (1971) has suggested that an instability due to a secondary peak in the electron velocity distribution function $f\left(v_{\perp}\right)$ may initiate the $f_{D 1}$ resonances ( $v_{\perp}$ is the component of the electron velocity perpendicular to $\vec{B}$ ). This model, however, does not explain the observed variation of $f_{D 1}$ as the plasma parameter $f_{N} / f_{H}$ changes.

The splitting of the $f_{D 1}$ resonance in high latitudes (see Figure 1a) is here interpreted in terms of a Doppler effect caused by the motion of the satellite. The energy contained in the $100 \mu$ sec sounder pulse propagates in all directions (see Figure $1 b$, time $t_{1}$ ), and some of this energy gives rise to a plasma instability. Since energy propagates in all directions, some wave components experience negligible Doppler shift; thus the center portion of the resonance is "filled in" during the short time delay portion of the $f_{D 1}$ resonance $\left(t_{1}\right.$ of Figure 1a). Due to cyclotron and Landau damping, only the energy propagating nearly perpendicular to $\vec{B}$ can reach great distances from the satellite [Tataronis and Crawford, 1966 ]. As this energy is absorbed into the medium, a turbulent region is produced, a nonlinear wave-wave resonant interaction is set up, and plasma waves (including waves at the frequency $f_{D 1}$ ) are re-radiated. Now, 
however, only the energy propagating nearly perpendicular to $\vec{B}$ can return to the satellite, i.e., the energy traveling along the return path of the wave packet that produced the turbulent region. Thus, all the received energy at this time delay ( $t_{2}$ of Figure 1$)$ experiences a Doppler shift. The original wave packet continues to give up energy to the medium as it propagates in the direction perpendicular to $\vec{B}$ (creating a cloud of turbulence along the way) until it no longer contains sufficient energy to produce a turbulent plasma. This point in time $\left(t_{3}\right.$ of Figure 1) corresponds to the longest duration portion of the $f_{D 1}$ resonance. Since the group velocity $v_{g}$ for the $f_{D 1}$ waves is of the order of $100 \mathrm{~m} / \mathrm{msec}$, the distance to the final turbulent region is approximately $100\left(t_{3} / 2\right) \mathrm{m}$ when $t_{3}$ is measured in msec. At greater distances there is no generation of $f_{D 1}$ waves, due to the lack of turbulence, and no signals are received at the corresponding delay times (such as $t_{4}$ in Figure 1).

Since the observations indicate that the original instability initiated by the sounder pulse in the vicinity of the satellite persists for no longer than $1.5 \mathrm{msec}$, and that the sounder pulse does not produce an instability beyond the distance $\mathrm{v}_{\mathrm{g}}\left(\mathrm{t}_{3} / 2\right)$ of Figure $1 \mathrm{~b}$ (typically $300 \mathrm{~m}$ ), it will be assumed that after a delay time of several milliseconds, e.g., $t_{2}$ of Figure $1 \mathrm{a}$, that the electrons in the propagation region between the sounder receiver and the source region for the $f_{D 1}$ waves have a Maxwellian distribution in $v_{\perp}$. Then the Bernstein (1958) dispersion equation for electrostatic waves propagating perpendicular to $\vec{B}$ can be used; it is convenient to represent this equation in the form of the curves of Figure 2 where $k$ is the absolute value of the propagation vector $\vec{k}$ and $R=\left[\kappa\left(T_{e}\right)_{\perp}\right]$ $\left.m\left(2 \pi f_{H}\right)^{2}\right]^{1 / 2}$. The wave number $k$ can be determined from the observed Doppler 
shift of the $f_{D 1}$ resonance since each portion of the resonance is shifted by $(1 / 2 \pi) \vec{k} \cdot \vec{V}_{\text {sat }}=(1 / 2 \pi) \mathrm{k}\left(\mathrm{V}_{\text {sat }}\right)_{\perp}$ for the Bernstein mode, where $\left(\mathrm{V}_{\text {sat }}\right)_{\perp}$ is the component of the satellite velocity $V_{\text {sat }}$ perpendicular to $\vec{B}$. The frequency difference $\Delta f$ between the two components of the $f_{D 1}$ resonance is just twice this value, thus $\left(\mathrm{T}_{\mathrm{e}}\right)_{\perp}$ can be expressed in terms of $\mathrm{kR}$ as follows:

$$
\left(\mathrm{T}_{\mathrm{e}}\right)_{\perp}=\left(\frac{\mathrm{m}}{\kappa}\right)\left(\frac{2\left(\mathrm{~V}_{\mathrm{sat}}\right)_{\perp} \mathrm{f}_{\mathrm{H}}}{\Delta \mathrm{f}}\right)^{2}(\mathrm{kR})^{2} .
$$

The quantity $k R$ can be obtained from Figure 2 (after the center frequency $f_{D 1}$, $f_{N}$, and $f_{H}$ have been scaled from the ionogram), $\left(V_{\text {sat }}\right)_{\perp}$ can be obtained from the satellite orbital information, and $\Delta \mathrm{f}$ is obtained from the ionogram.

Alouette II diffuse resonances were scaled from the conventional ionogram format using a linear interpolation between frequency markers and applying frequency corrections for the non-linear frequency sweep rate of the Alouette II sounder [Benson, 1970]. A frequency correction of $-10 \mathrm{kHz}$ was applied to the center frequency $f_{D 1}$ (and to other resonances that did not reveal the frequency spectrum of the transmitted pulse) in order to compensate for the $-10 \mathrm{kHz}$ offset of the receiver with respect to the transmitter [Benson, 1969]; a frequency correction of $-6 \pm 2 \mathrm{kHz}$ was also applied to the scaled frequencies in order to compensate for the positive offset of the Alouette II transmitter [Benson, 1972]. The sharp upper frequency cut-off of each Doppler shifted component of the $f_{D 1}$ resonance was scaled in order to obtain $\Delta f$; these cut-offs correspond to the receivers $3 \mathrm{~dB}$ points. The effect of the rotating earth was considered in the determination of $\left(\mathrm{V}_{\text {sat }}\right)_{\perp}$. A value for $\left(\mathrm{T}_{\mathrm{e}}\right)_{\perp}$ was then obtained from (1).

Only data corresponding to times when the Alouette II electrostatic probe experiment was in operation were considered. The results are presented in Figures 3 and 4 for data recorded at the Ottawa and Winkfield telemetry 
stations. The electron temperature values obtained from the $f_{D 1}$ resonance $\left(T_{e}\right)_{f_{D 1}}$ data are consistently below the values obtained from the probe experiment $\left(\mathrm{T}_{\mathrm{e}}\right)$ probe in the Ottawa region (e.g., the average value of $\left(\mathrm{T}_{\mathrm{e}}\right)_{\text {probe }} /\left(\mathrm{T}_{\mathrm{e}}\right)_{\mathrm{f}_{\mathrm{D} 1}}$ in Figure $4 \mathrm{a}$ is 1.32 with a standard deviation from the mean $\sigma / \sqrt{n}=0.04)$, whereas the values are in agreement in the Winkfield region $\left(\mathrm{e}_{\bullet} \mathrm{g}_{\bullet}\right.$, the average value of $\left(\mathrm{T}_{\mathrm{e}}\right)_{\text {probe }} /$ $\left(\mathrm{T}_{\mathrm{e}}\right)_{\mathrm{f}_{\mathrm{D} 1}}$ in Figure $4 \mathrm{~b}$ is 1.02 with a standard deviation from the mean of 0.04$)$. The result, i.e., $\left(\mathrm{T}_{\mathrm{e}}\right)_{\mathrm{f}_{\mathrm{D} 1}} \leqslant\left(\mathrm{~T}_{\mathrm{e}}\right)_{\text {probe }}$ supports the assumption that the artificially stimulated $\left(\mathrm{T}_{\mathrm{e}}\right)_{\perp}$ value is not maintained in the propagation region after a time delay of a few milliseconds since $\left(T_{e}\right)_{f_{D 1}}$ corresponds to $\left(T_{e}\right)_{\perp}$ whereas $\left(T_{e}\right)_{\text {probe }}$ is not considered to be sensitive to direction.

The temperature discrepancy observed near Ottawa, but not near Winkfield, appears to be of geophysical rather than instrumental origin since two independent in-situ methods for determining $\mathrm{T}_{\mathrm{e}}$ from the same satellite are involved. This conclusion supports the recent experiments of Brace et al. (1971), where simultaneous rocket and satellite measurements were found to agree, and Brace and McClure (1971) which suggest a geophysical cause for the discrepancy. The present observations are consistent with a recent comparison by Taylor and Wren (1970) of electron temperatures obtained from the Explorer 31 Langmuir plate experiment and radar backscatter observations in the Winkfield region; they found a reasonable agreement between the two methods.

Since the diffuse resonance is mainly dependent on the electrons of lowest energy (it is due to a collective phenomena in the plasma which is not very dependent on the electrons at the high energy end of the energy distribution function), as is the radar backscatter signal (Hoegy, 1971), the present observations 
support one of the suggestions made by Hoegy (1971) - namely, that the discrepancy may be due to a departure of the low energy ionospheric electrons from a Maxwellian distribution.

\section{Acknowledgements}

We are grateful to Mr. L. H. Brace for supplying electron temperature values from his electrostatic probe experiment on Alouette II and for many informative discussions, and to Dr. S. J. Bauer for many helpful comments.

Part of this work was completed while one of us (H. O.) was a NAS-NRC Postdoctoral Resident Research Associate at the Goddard Space Flight Center. 


\section{References}

Brace, L. H., H. C. Carlson, and K. K. Mahajan, Radar backscatter and rocket probe measurements of electron temperature above Arecibo, J. Geophys. Res., 74, 1883-1885, 1969.

Brace, L. H. and J. P. McClure, Comparisons of equatorial rocket probe and radar observations (abstract), Eos Trans. AGU, 52, 290, 1971.

Brace, L. H., G. R. Carignan, and J. A. Findlay, Evaluation of ionospheric electron temperature measurements by cylindrical electrostatic probes, Space Res. 11, 1080-1105, 1971.

Benson, R. F., Frequency shifts observed in the Alouette 2 cyclotron harmonic plasma resonances, Proc. IEEE, 57, 1139-1142, 1969.

Benson, R. F., Frequency interpolation correction for Alouette II ionograms, Proc. IEEE, 58, 1959-1960, 1970.

Benson, R. F., Frequency shifts of ionospheric $\mathrm{nf}_{\mathrm{H}}$ resonances, to be published in J. Atmospheric Terrest. Phys., 1972.

Bernstein, I. B., Waves in a plasma in a magnetic field, Phys. Rev., 109, $10-21,1958$.

Fredricks, R. W., Plasma instability at $(n+1 / 2) f_{c}$ and its relationship to some satellite observations, J. Geophys. Res., 76, 5344-5348, 1971.

Hanson, W. B., L. H. Brace, P. L. Dyson, and J. P. McClure, Conflicting electron temperature measurements in the upper $\mathrm{F}$ region, J. Geophys. Res., 74, 400-401, 1969.

Hoegy, W. R., Probe and radar electron temperatures in an isotropic nonequilibrium plasma, J. Geophys. Res., 76, 8333-8340, 1971. 
Oya, H., Sequence of diffuse plasma resonances observed on Alouette 2 ionograms, J. Geophys. Res., 75, 4279-4285, 1970.

Oya, H., Verification of theory on weak turbulence relating to the sequence of diffuse plasma resonances in space, Phys. Fluids, 14, 2487-2499, 1971.

Tataronis, J. A., and F. W. Crawford, Cyclotron and collision damping of propagating waves in a magnetoplasma, Proceedings of the 7th International Conference on Phenomena in Ionized Gases, Belgrad, Aug. 1965 II, pp. 244-247 Ed. by B. Perović and D. Tošić, Gradevinska Knjiga Pub. House, Belgrad, 1966.

Taylor, G. N., and G. L. Wrenn, Comparisons of simultaneous satellite and ground-based measurements of ionospheric parameters, Planet. Space Sci., 18, 1663-1666, 1970.

Warnock, J. M., J. R. McAfee, and T. L. Thompson, Electron temperature from topside plasma resonance observations, J. Geophys. Res., 75, 72727275,1970 . 


\section{Figure Captions}

Figure 1 Example of the splitting of the $f_{D 1}$ resonance (a) and a sketch of the model proposed to explain the observations (b). The sketch shows four "snap shots" of the medium in the vicinity of the satellite at times $t_{1}, t_{2}, t_{3}$, and $t_{4}$ after the transmission of the sounder pulse. These times are also identified on the data insert in (a); the time $t_{3}$ corresponds to $6 \mathrm{msec}$. The $\mathrm{f}_{\mathrm{D} 1}$ resonance in (a) was taken from an Alouette 2 Ottawa ionogram recorded at 21:43:50 UT on pass 1775 (27 April 1966).

Figure 2 Dispersion curves for the Bernstein mode, i.e., propagation perpendicular to $\vec{B}$, in the frequency domain of the $f_{D 1}$ resonance.

Figure 3 Comparison of simultaneous in-situ electron temperature measurements using the Alouette 2 diffuse resonance data (solid points) and the Alouette 2 electrostatic probe data (open circles) for two passes over Ottawa ( $a$ and $b$ ) and one pass over Winkfield (c). The corresponding values for the electron density $\mathrm{N}$ (as obtained from the plasma resonance data) and the satellite altitude $\mathrm{z}$ are given at the top of the figure. The data, in all three cases, were obtained between 23 and 26 April 1966 and between 16 and 17 hours local time (ionogram times between 20:49:46 to 20:52:13 UT from OTT pass 1739 in (a), 21:25:13 to $21: 26: 48$ UT from OTT pass 1763 in (b) and, 16:34:21 to $16: 35: 55$ UT from WNK pass 1725 in (c)).

Figure 4 Similar electron temperature comparison (as in Figure 3) for several passes through a small region of space near Ottawa (a) and a similar 
region near Winkfield (b). The data points correspond to the time when the $f_{D 1}$ resonance was recorded (this time is usually between 6 and 8 seconds after the printed ionogram time). The geographic dimensions of the data regions are as follows: Ottawa (a) 32.4 to $38.7^{\circ} \mathrm{N}$ latitude, 66.2 to $85.5^{\circ} \mathrm{W}$ longitude, 510 to $538 \mathrm{~km}$; Winkfield (b) 48.6 to $54.8^{\circ} \mathrm{N}$ latitude, $17.0^{\circ} \mathrm{E}$ to $1.8^{\circ} \mathrm{W}$ longitude, 511 to $534 \mathrm{~km}$. Three of the data point pairs from Figure 3(a and b) are included in (a) of the present figure; the four data point pairs from Figure 3(c) are included in (b) of the present figure. 

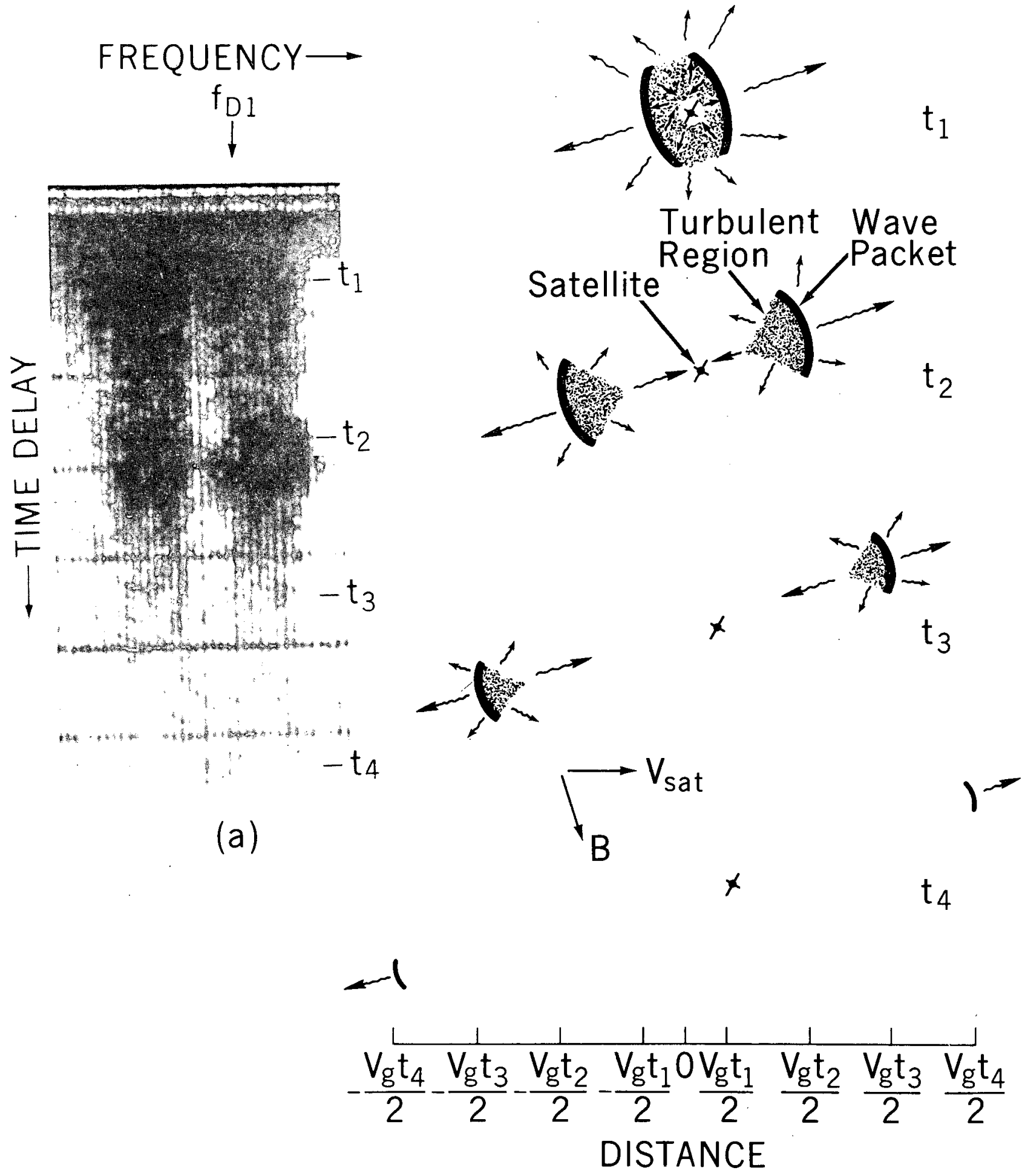

(b) 


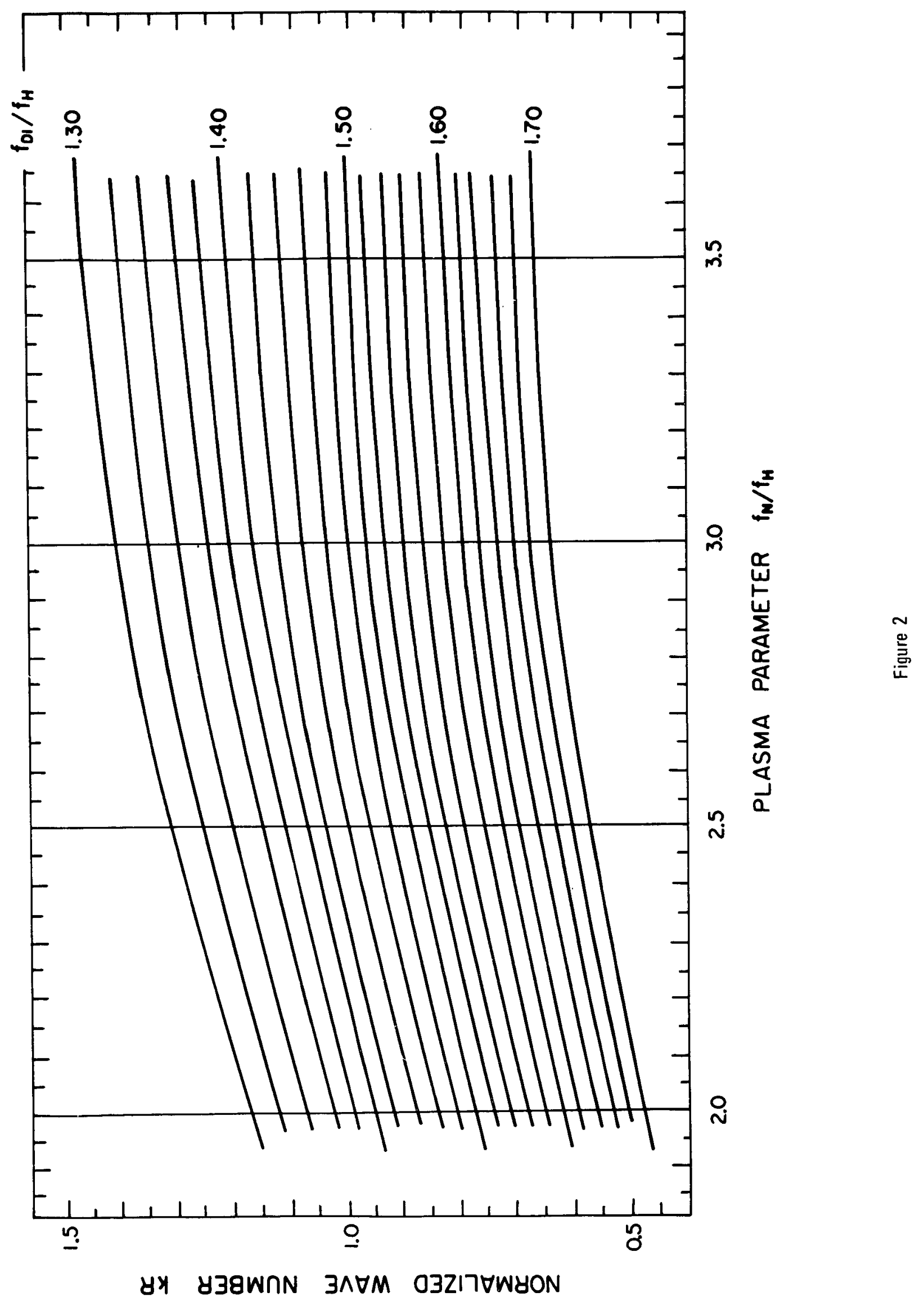




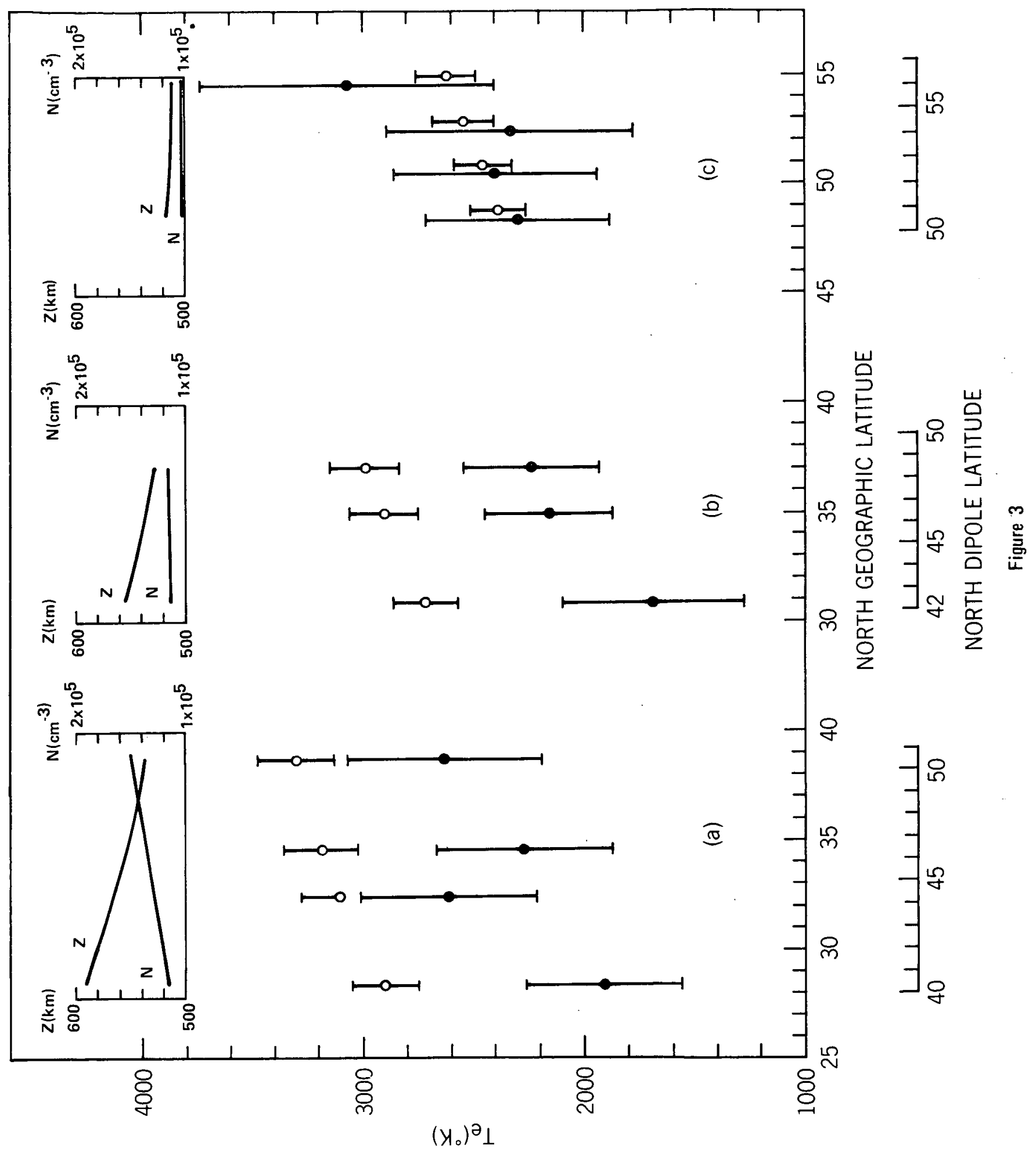




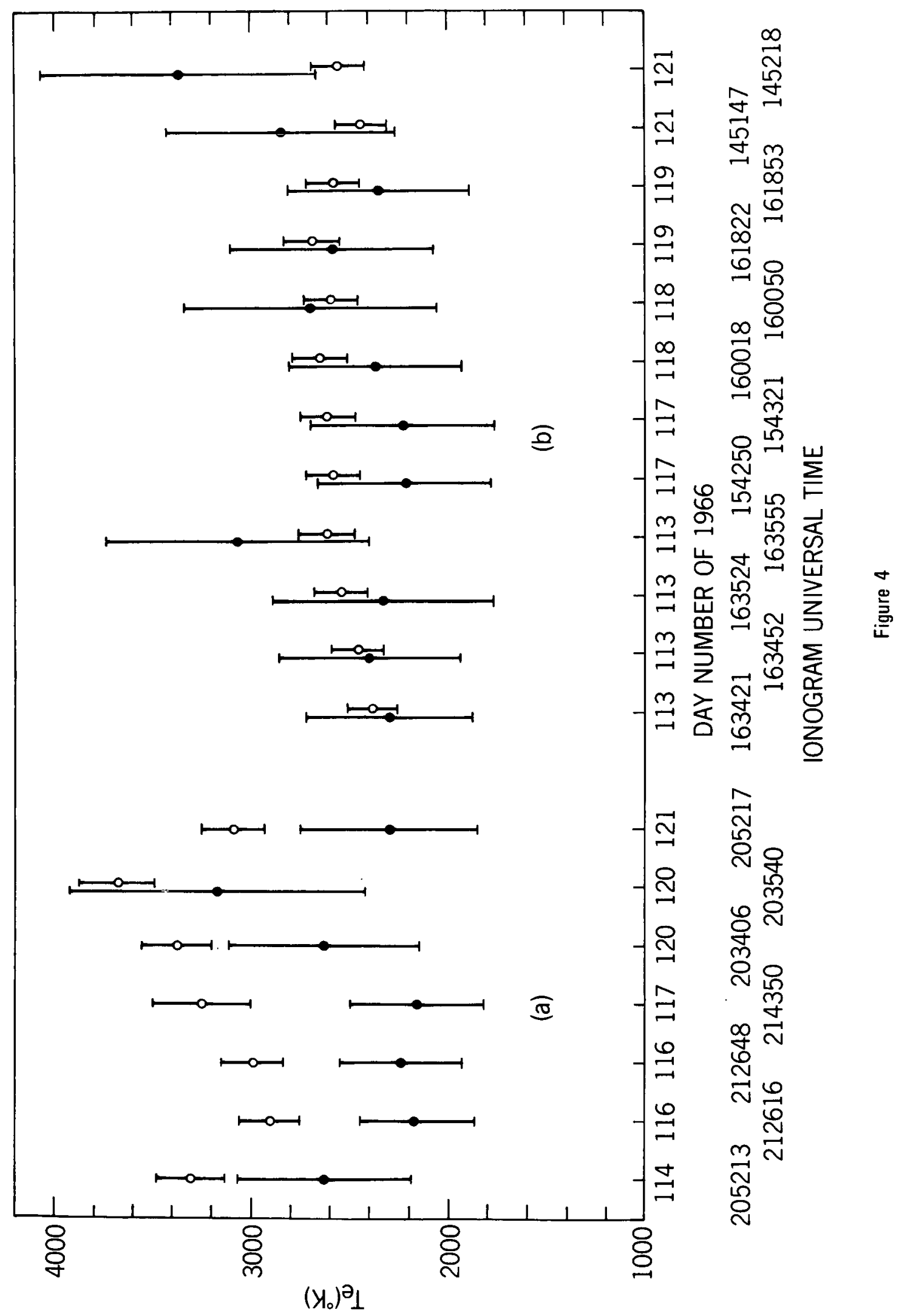

